\title{
INFLUENCE \\ DE LA TEMPÉRATURE DE CALCINATION SUR LA DÉTERMINATION MESURÉE DE QUELQUES RADIOÉLÉMENTS CONTENUS DANS LES PRÉLĖVEMENTS ATMOSPHÉRIQUES
}

\author{
(manuscrit rę̧u le i er décembre I966) $^{2}$
}

Les méthodes de collecte des contaminants atmosphériques nécessitent une séparation de la partie soluble dans les prélèvements provenant des précipitations. Il n'en est pas de même des prélèvements "secs" de poussières réalisés soit par aspiration sur filtres, soit par fixation des particules sur des matières adhésives.

Dans les prélèvements atmosphériques en provenance de précipitations, nous séparons arbitrairement la partie "soluble » et la partie « insoluble » en effectuant la filtration à la température du laboratoire au $p \mathrm{H}$ mesuré de l'eau et sur un type de filtre donné [r]. Le prélèvement contient généralement des débris organiques, végétaux ou animaux (insectes) qui peuvent avoir, vis-à-vis des produits dissous dans l'eau, un comportement bien difficile à prévoir :

La partie insoluble est généralement calcinée.

Nous avons voulu connaitre quel était, pour quelques éléments radioactifs, le pourcentage de la radioactivité perdue au cours de la calcination.

$\mathrm{I}^{\circ}$ Nous avons calciné à $900^{\circ} \mathrm{C}$ un papier adhésif de "Codirat" [2] [3]. Nous avons noté des pertes très importantes en ${ }^{103} \mathrm{Ru},{ }^{106} \mathrm{Ru}+{ }^{106} \mathrm{Rh}$ et en ${ }^{137} \mathrm{Cs}$. Par contre il n'y a pas de perte en ${ }^{141} \mathrm{Ce}$ et en ${ }^{144} \mathrm{Ce}$ ainsi que le montrent les spectrogrammes I et 2 (fig. I).

Nous avons évaporé 4 solutions étalonnées de ${ }^{137} \mathrm{Cs}$. En chauffant au four dans les conditions de calcinations habituelles les quatre résidus secs pendant 30 minutes, nous constatons qu'à $500^{\circ} \mathrm{C}$ il n'y a aucune perte par calcination tandis qu'à $900^{\circ} \mathrm{C}$ les pertes se situent entre $8 \mathrm{I}$ et $89 \%$ de la concentration initiale.

$2^{\circ}$ Nous avons répété la même expérience avec les Zirconium-Niobium 95 dont le résidu sec a été chauffé en creuset pendant 6 heures à $500^{\circ}$. Nous constatons que ces éléments ne sont pas volatils et que le chauffage n'entraîne aucune perte dans l'analyse.

$\mathrm{Le}^{141} \mathrm{Ce}$ est très stable si on le chauffe à $500^{\circ} \mathrm{C}$ et la petite différence que nous avons observée $(0,5 \%)$ est de l'ordre de grandeur des erreurs de mesures de nos appareils.

Nous avons repris ces essais sur des résidus insolubles d'eau de pluie qui ont été calcinés à $500^{\circ} \mathrm{C}$ en creuset couvert pendant 6 heures.

Nous n'avons pas noté de perte en $\left({ }^{95} \mathrm{Zr}+{ }^{95} \mathrm{Nb}\right)$ en Cérium 144 et en Césium 137 ce qui confirme les résultats de nos expériences.

Il faut remarquer que nous n'observons pas de perte en ruthénium. Le ruthénium présent dans les retombées se trouve dans un état physico-chimique tel qu'il n'est pas volatil à $500^{\circ} \mathrm{C}$.

Cette série d'expériences montre que la méthode de calcination des prélèvements doit être employée avec précaution pour la mesure de la radioactivité de solutions très diluées soit après séparation physico-chimique, soit en mesurant la radioactivité $\alpha \beta$ globale, soit la radioactivité des émetteurs $\gamma$ par spectrométrie.

A. CANET et H. FRANÇOIS

Service technique d'Etudes de Protection, Centre d'Etudes Nucléaires de Fontenay-auxRoses, B.P. $n^{\circ}$ 6, 92-Fontenay-aux-Roses. 


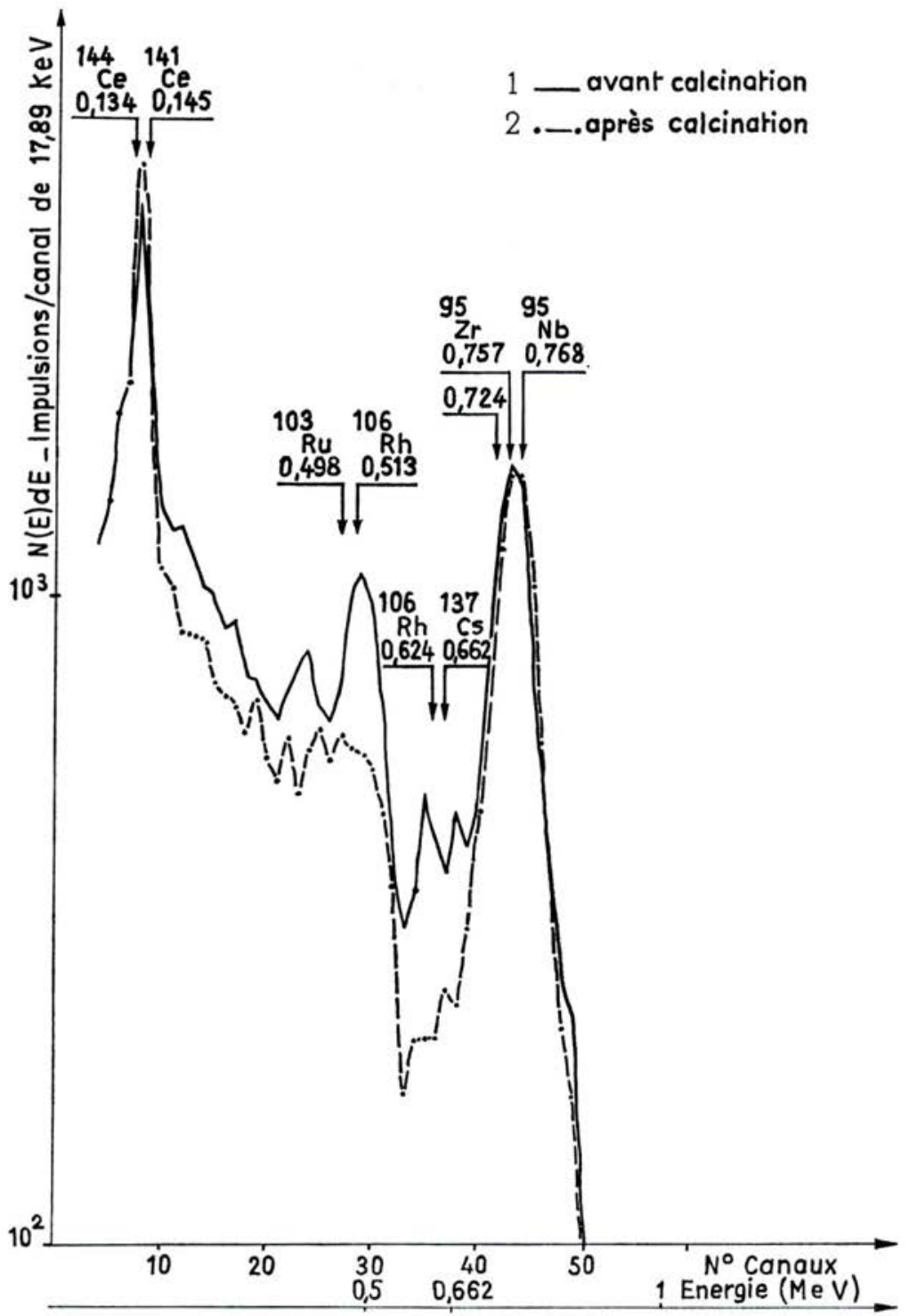

FIG. I. - Spectrogrammes $\gamma$ des retombées atmosphériques prélevées sur papier adhésif * Codirat ", avant et après calcination à $900^{\circ}$.

[i] François H., Leblond-Tourni A. et Canet A. - Compte rendu de la Conférence sur la pollution radioactive des milieux gazeux, Saclay $1_{96}$.

[2] Doury A., Françors H. et Blondeau E. - Brevet français no 887.544, 9 février 1962. 


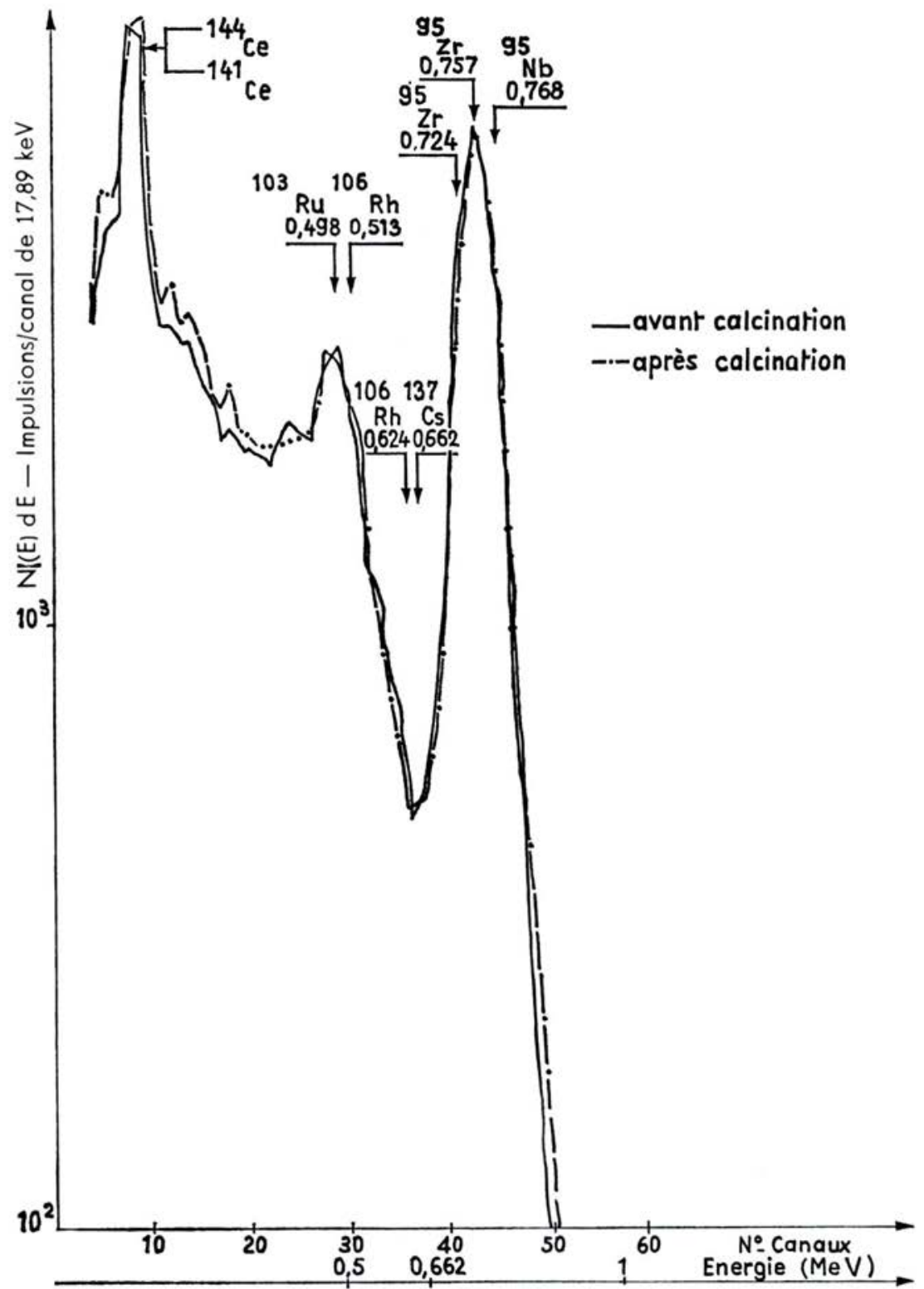

FIg. 2. - Etude de la calcination d'un résidu insoluble d'eau de pluie.

[3] Doury A, Françors H., Blondeau E. et Société ERCA à Clamart (Seine), Brevet français $\mathrm{n}^{\circ} 917.654$ du s décembre 1962. 